\title{
Genome Sequence Resource for llyonectria mors-panacis, Causing Rusty Root Rot of Panax notoginseng
}

\author{
Bo Zhu, ${ }^{1,2}$ Sai Wang, ${ }^{1,2}$ Chun-Yi Mi, ${ }^{1}$ Rui-Huan Yang, ${ }^{1}$ Guo-Hong Zen, ${ }^{1}$ and Xiu-Fang $\mathrm{Hu}^{1 \dagger}$ \\ ${ }^{1}$ Zhejiang Province Key Laboratory of Plant Secondary Metabolism and Regulation, College of Life \\ Science, Zhejiang Sci-Tech University, Road 2, 310018, Hangzhou, China \\ ${ }^{2}$ School of Agriculture and Biology, Shanghai Jiao Tong University/Key Laboratory of Urban Agriculture \\ by Ministry of Agriculture of China, 200240, Shanghai, China
}

\section{Abstract}

Ilyonectria mors-panacis is the cause of a serious disease hampering the production of Panax notoginseng, an important Chinese medicinal herb, widely used for its anti-inflammatory, antifatigue, hepato-protective, and coronary heart disease prevention effects. Here, we report the first Illumina-Pacbio hybrid sequenced draft genome assembly of $I$. mors-panacis strain G3B and its annotation. The availability of this genome sequence not only represents an important tool toward understanding the genetics behind the infection mechanism of $I$. morspanacis strain G3B but also will help illuminate the complexities of the taxonomy of this species.

\section{Genome Announcement}

Ilyonectria mors-panacis is the pathogen inducing the severe rusty root rot of Panax notoginseng, an important traditional Chinese medicinal herb (Hong et al. 2014; Mi et al. 2017), which is widely used for its anti-inflammatory, antifatigue, hepato-protective, and coronary heart disease prevention effects (Li et al. 2007; Xia et al. 2016). I. mors-panacis was grouped in the genus Cylindrocarpon, which is commonly associated with rot and decay of woody and herbaceous plants (Rahman and Punja 2005). Mantiri et al. (2001) estimated that there are approximately 125 described species of Cylindrocarpon and most of them have been classified into the genus Neonectria (Mantiri et al. 2001). Based on phylogenetic and phenotypical characteristics, Chaverri et al. (2011) redefined Neonectria into four genera: anamorph Ilyonectria (Booth's group 3) with chlamydospores, teleomorph genera Neonectria or Cylindrocarpon sensu stricto (groups 1 and 4), and Rugonectria and Thelonectria (group 2) without chlamydospores (Chaverri et al. 2011). Recently, Cabral et al. (2012) renamed the Nectria or Neonectria radicicola (including Cylindrocarpon destructans) complex as the Ilyonectria radicicola complex, and reclassified the fungi in this complex into 15 species under the genus llyonectria. In particular, highly virulent $I$. radicicola isolates of $P$. ginseng (e.g.,

\section{${ }^{\dagger}$ Corresponding author: X.-F. Hu; huxiuf@zstu.edu.cn}

*The e-Xtra logo stands for "electronic extra" and indicates that one supplementary figure and one supplementary table are published online.

The author(s) declare no conflict of interest.

Accepted for publication 1 July 2019.

\section{Funding}

The research was supported by National Key R\&D Program of China (2017YFD0201108 and 2018YFD0201202), Zhejiang Natural Science Foundation of China (LY16C030002 and LY17C010006), National Natural Science Foundation of China (81673537), Key Research and Development Projects of Zhejiang Province (2015C02030), Zhejiang Province Public Agricultural Project (2014C32117), the Agri-X Interdisciplinary Fund of Shanghai Jiao Tong University (Agri-X2017010) and State Key Laboratory for Biology of Plant Diseases and Insect Pests (SKLOF201802).

\section{Keywords}

genomics, Ilyonectria mors-panacis, metabolomics, Pacbio,

Panax notoginseng, pathogenesis, proteomics 
IFO31881, IFO31882, NSAC SH1, and NSAC SH2.5) were included in I. mors-panacis (Song et al. 2014). Until now, only one strain from I. radicicola complex was partially sequenced (>3,000 contigs). Due to its impact as a traditional Chinese medicinal herb and its complex taxonomic classification, I. mors-panacis is a good candidate to carry out whole-genome sequencing.

I. mors-panacis strain G3B was first isolated from $P$. notoginseng (Burk.) F. H. Chen in 2014 from Wenshan, Yunnan Province, China (Mi et al. 2017). The strain was maintained on potato dextrose agar. It was subcultured in potato dextrose broth at $22^{\circ} \mathrm{C}$ for 5 days at $180 \mathrm{rpm}$. High-quality genomic DNA was extracted from $50 \mathrm{ml}$ of culture, using the Plant/ Fungi DNA Isolation Kit (Sigma-Aldrich). To confirm the ploidy of the G3B genome, Jellyfish V 2.2.10 was used to calculate the k-mer distribution from the reads (Marçais and Kingsford 2011). The result showed that the k-mer curve of G3B is significantly a single peak without a heterozygous peak (Supplementary Fig. S1). Based on the above plotting, we confirmed that the sequenced genome is a haploid. The quality of the extracted DNA was tested with a Qubit 2.0 fluorometer (Life Technologies) and a NanoDrop 2000 UV-Vis spectrophotometer (Thermo Scientific). For Pacbio sequencing, after genomic DNA shearing, $1.5 \mu \mathrm{g}$ of the sheared DNA was used to prepare an SMRTbell library with the PacBio SMRTbell Template Prep Kit 1 (Pacific Biosciences). Sequence runs for one single-molecule real-time (SMRT) cell were performed on the PacBio RS II sequencer with a 120-min movie time per SMRT cell. After quality control, 321,858 reads were used for further assembly. For Illumina sequencing, the library was prepared using the TruSeq Nano DNA LT Library Preparation Kit (Illumina) using $250 \mathrm{ng}$ of genomic DNA. The resulting library was $150 \mathrm{bp}$ paired-end sequenced on the Illumina HiSeq 2500. After quality control, 23,449,680 reads were used for further assembly. The I. mors-panacis G3B genome was de novo assembled by using the Hierarchical Genome Assembly Process, which can be accessed through the SMRT Analysis Portal. PBJelly v14.1.14 was used to fill and reduce as many captured gaps as possible, and produce a draft genome (English et al. 2012) by using Illumina sequencing reads. Last, Illumina reads were used to repair the sequencing errors generated by SMRT sequencing. The completeness of assembly was evaluated using the software BUSCO v3 with a set of common fungal singlecopy orthologs (Waterhouse et al. 2017). This result showed that the G3B genome assembly is estimated to be $96.8 \%$ complete. The genome annotation was done with Augustus V3.3.1

Table 1. Core metrics of the Ilyonectria mors-panacis G3B genome draft and ANI comparison between I. mors-panacis G3B and other closely related fungi genomes

\begin{tabular}{|c|c|c|c|c|c|}
\hline Metrics and comparisons ${ }^{a}$ & Value & Strain & Accession number & $\begin{array}{l}\text { ANI-NUCmer } \\
\text { values }\end{array}$ & $\begin{array}{c}\text { ANI-Blast } \\
\text { values }\end{array}$ \\
\hline \multicolumn{6}{|l|}{ General genome characteristic } \\
\hline Assembly size $(\mathrm{bp})$ & $61,489,895$ & $\ldots$ & $\ldots$ & $\cdots$ & $\cdots$ \\
\hline Contig number & 145 & $\ldots$ & $\ldots$ & $\ldots$ & $\ldots$ \\
\hline Maximum contig size (bp) & $2,825,696$ & $\ldots$ & $\ldots$ & $\ldots$ & $\ldots$ \\
\hline Minimum scaffold size (bp) & 12,370 & $\ldots$ & $\ldots$ & $\ldots$ & $\ldots$ \\
\hline Average scaffold size (bp) & 424,068 & $\ldots$ & $\ldots$ & $\ldots$ & $\ldots$ \\
\hline $\mathrm{N}_{50}$ length $(\mathrm{bp})$ & $1,010,205$ & $\ldots$ & $\ldots$ & $\cdots$ & $\cdots$ \\
\hline $\mathrm{N}_{90}$ length (bp) & 290,231 & $\ldots$ & $\ldots$ & $\ldots$ & $\ldots$ \\
\hline $\mathrm{G}+\mathrm{C}(\%)$ & 48.7 & $\ldots$ & $\ldots$ & $\ldots$ & $\ldots$ \\
\hline Intron number & 45,382 & $\ldots$ & $\ldots$ & $\ldots$ & $\ldots$ \\
\hline Intron density & 1,355 & $\ldots$ & $\ldots$ & $\ldots$ & $\ldots$ \\
\hline Gene contains intron & 13,594 & $\ldots$ & $\ldots$ & $\ldots$ & $\ldots$ \\
\hline PCG & 18,321 & $\ldots$ & $\ldots$ & $\ldots$ & $\ldots$ \\
\hline PCG > 100 aa & 17,585 & $\cdots$ & $\cdots$ & $\cdots$ & $\cdots$ \\
\hline \multicolumn{6}{|l|}{ Predicted PCG > 100 aa } \\
\hline Gene total length (bp) & $26,931,632$ & $\ldots$ & $\ldots$ & $\cdots$ & $\cdots$ \\
\hline Gene density & 3,496 & $\ldots$ & $\ldots$ & $\cdots$ & $\cdots$ \\
\hline Median gene length (bp) & 1,239 & $\ldots$ & $\ldots$ & $\ldots$ & $\ldots$ \\
\hline Mean gene length (bp) & 1,531 & $\ldots$ & $\ldots$ & $\ldots$ & $\ldots$ \\
\hline $\mathrm{G}+\mathrm{C}(\%)$ & 51.9 & $\cdots$ & $\ldots$ & $\cdots$ & $\ldots$ \\
\hline \multicolumn{6}{|l|}{ ANI comparison (species) } \\
\hline Ilyonectria mors-panacis & $\ldots$ & G3B & PPHJ00000000 & 1.000 & 1.000 \\
\hline Dactylonectria macrodidyma & $\ldots$ & JAC 15-245 & JYGD00000000 & 0.852 & 0.787 \\
\hline Neonectria ditissima & $\ldots$ & RS 324p & LDPL01000040.1 & 0.846 & 0.780 \\
\hline Calonectria pseudonaviculata & $\ldots$ & CBS 139394 & JYJY00000000 & 0.842 & 0.747 \\
\hline Fusarium solani & $\ldots$ & JS-169 & NGZQ00000000 & 0.745 & 0.732 \\
\hline Ilyonectria destructans & $\ldots$ & $\mathrm{C} 1$ & MPHF00000000 & 0.915 & 0.906 \\
\hline
\end{tabular}

\footnotetext{
a PCG $=$ protein coding genes, aa $=$ amino acid, and intron and gene density $=1$ gene every $n$ bp.
} 
(Stanke and Morgenstern 2005) and WebAUGUSTUS, a web interface, was used for training Augustus (Hoff and Stanke 2013). The training was performed by using it on its own genome sequence and the gene model of Fusarium oxysporumf. sp. Iycopersici 4287 (Ma et al. 2010) obtained from the Joint Genome Institute. Additional analysis was carried out using NCBl's UniProt database, Kyoto Encyclopedia of Genes and Genomes (Kanehisa and Goto 2000), and Gene Ontology terms (Ashburner et al. 2000). Virulence genes were annotated by using the Database of Virulence Factors in Fungal Pathogens (DFVF) (Lu et al. 2012). Genome comparison among G3B and other closely related genomes was carried out by using average nucleotide identity (ANI), which was calculated by ANIcalculator (Varghese et al. 2015).

After assembly, this genome contained 145 contigs with $N_{50}$ of $1,010,205 \mathrm{bp}$. The total size of the genome is $61,489,751 \mathrm{bp}$ and it has a GC content of $48.72 \%$. In total, 18,321 coding sequences were predicted. Additional features of this genome are shown in Table 1. To access the similarity among G3B and other closely related organisms, all available sequenced fungal genomes from the family Nectriaceae were downloaded from NCBI and compared against G3B. Strikingly, we found low ANI between G3B and the other closely related organisms (Table 1). Even the ANI between I. mors-panacis G3B and I. destructans C1 is only $90.27 \%$ (Table 1).

Overall, the annotation analysis predicted 120 genes defined by DFVF with involvement in pathogenesis (Supplementary Table S1): 28 as cell-wall-degrading enzymes, which include cellulases, $\beta$-glucosidase, endo- $\beta-1,4$-xylanase, pectinases, pectin methylestrase, and laccase; 18 as antioxidant systems, which include superoxide dismutase and carotenoid; and, finally, 7 defined as effectors.

The draft genome presented here is the first available genome sequence of $I$. morspanacis, and only the second from the genus llyonectria. Because these are causal organisms leading to $P$. notoginseng replanting problems, understanding the whole genome of I. mors-panacis G3B will be important for developing suitable root rot disease management strategies in the future. This Whole-Genome Shotgun project has been deposited at GenBank under the accession number PPHJ00000000.1(BioProject: PRJNA431033). The version described in this article is version PPHJ00000000.1.

\section{Author-Recommended Internet Resources}

Joint Genome Institute: https://genome.jgi.doe.gov/Fusox2/Fusox2.home.html

NCBl's UniProt database: https://www.ncbi.nlm.nih.gov/

\section{Literature Cited}

Ashburner, M., Ball, C. A., Blake, J. A., Botstein, D., Butler, H., Cherry, J. M., Davis, A. P., Dolinski, K., Dwight, S. S., Eppig, J. T., Harris, M. A., Hill, D. P., Issel-Tarver, L., Kasarskis, A., Lewis, S., Matese, J. C., Richardson, J. E., Ringwald, M., Rubin, G. M., and Sherlock, G.; The Gene Ontology Consortium. 2000. Gene ontology: Tool for the unification of biology. Nat. Genet. 25:25-29.

Cabral, A., Groenewald, J. Z., Rego, C., Oliveira, H., and Crous, P. W. 2012. Cylindrocarpon root rot: Multi-gene analysis reveals novel species within the Ilyonectria radicicola species complex. Mycol. Prog. 11:655-88.

Chaveri, P., Salgado, C., Hirooka, Y., Rossman, A. Y., and Samuels, G. J. 2011. Delimitation of Neonectria and Cylindrocarpon (Nectriaceae, Hypocreales, Ascomycota) and related genera with Cylindrocarpon-like anamorphs. Stud. Mycol. 68:57-78.

English, A. C., Richards, S., Han, Y., Wang, M., Vee, V., Qu, J., Qin, X., Muzny, D. M., Reid, J. G., Worley, K. C., and Gibbs, R. A. 2012. Mind the gap: Upgrading genomes with Pacific Biosciences RS long-read sequencing technology. PLoS One 7: e47768.

Hoff, K. J., and Stanke, M. 2013. WebAUGUSTUS-A web service for training AUGUSTUS and predicting genes in eukaryotes. Nucleic Acids Res. 41:W123-W128.

Hong, J., Hu, J.-Y., Liu, J.-H., Zhou, Z., and Zhao, A.-F. 2014. In vitro antioxidant and antimicrobial activities of flavonoids from Panax notoginseng flowers. Nat. Prod. Res. 28:1260-1266.

Kanehisa, M., and Goto, S. 2000. KEGG: Kyoto Encyclopedia of Genes and Genomes. Nucleic Acids Res. 28:27-30.

Li, X., Wang, G., Sun, J., Hao, H., Xiong, Y., Yan, B., Zheng, Y., and Sheng, L. 2007. Pharmacokinetic and absolute bioavailability study of total Panax notoginsenoside, a typical multiple constituent traditional Chinese medicine (TCM) in rats. Biol. Pharm. Bull. 30:847-851.
Lu, T., Yao, B., and Zhang, C. 2012. DFVF: Database of fungal virulence factors. Database (Oxford) 2012:bas032.

Ma, L.-J., van der Does, H. C., Borkovich, K. A., Coleman, J. J., Daboussi, M.-J., Di Pietro, A., Dufresne, M., Freitag, M., Grabherr, M., Henrissat, B., Houterman, P. M., Kang, S., Shim, W. B., Woloshuk, C., Xie, X., Xu, J. R., Antoniw, J., Baker, S. E., Bluhm, B. H., Breakspear, A., Brown, D. W., Butchko, R. A., Chapman, S., Coulson, R., Coutinho, P. M., Danchin, E. G., Diener, A., Gale, L. R., Gardiner, D. M., Goff, S., Hammond-Kosack, K. E., Hilburn, K., Hua-Van, A., Jonkers, W., Kazan, K., Kodira, C. D., Koehrsen, M., Kumar, L., Lee, Y. H., Li, L., Manners, J. M., Miranda-Saavedra, D., Mukherjee, M., Park, G., Park, J., Park, S. Y., Proctor, R. H., Regev, A., Ruiz-Roldan, M. C., Sain, D., Sakthikumar, S., Sykes, S., Schwartz, D. C., Turgeon, B. G., Wapinski, I., Yoder, O., Young, S., Zeng, Q., Zhou, S., Galagan, J., Cuomo, C. A., Kistler, H. C., and Rep, M. 2010. Comparative genomics reveals mobile pathogenicity chromosomes in Fusarium. Nature 464:367-373.

Mantiri, F. R., Samuels, G. J., Rahe, J. E., and Honda, B. M. 2001. Phylogenetic relationships in Neonectria species having Cylindrocarpon anamorphs inferred from mitochondrial ribosomal DNA sequences. Can. J. Bot. 79: 334-340.

Marçais, G., and Kingsford, C. 2011. A fast, lock-free approach for efficient parallel counting of occurrences of k-mers. Bioinformatics 27:764-770.

Mi, C., Yang, R., Rao, J., Yang, S., Wei, F., Li, O., and Hu, X. 2017. Unveiling of dominant fungal pathogens associated with rusty root rot of Panax notoginseng based on multiple methods. Plant Dis. 101:2046-2052.

Rahman, M., and Punja, Z. K. 2005. Factors influencing development of root rot on ginseng caused by Cylindrocarpon destructans. Phytopathology 95: 1381-1390. 
Song, J. Y., Seo, M. W., Kim, S. I., Nam, M. H., Lim, H. S., and Kim, H. G. 2014. Genetic diversity and pathogenicity of Cylindrocarpon destructans isolates obtained from Korean Panax ginseng. Mycobiology 42:174-180.

Stanke, M., and Morgenstern, B. 2005. AUGUSTUS: A web server for gene prediction in eukaryotes that allows user-defined constraints. Nucleic Acids Res. 33: W465-W467.

Varghese, N. J., Mukherjee, S., Ivanova, N., Konstantinidis, K. T., Mavrommatis, K., Kyrpides, N. C., and Pati, A. 2015. Microbial species delineation using whole genome sequences. Nucleic Acids Res. 43:6761-6771.
Waterhouse, R. M., Seppey, M., Simão, F. A., Manni, M., Ioannidis, P., Klioutchnikov, G., Kriventseva, E. V., and Zdobnov, E. M. 2017. BUSCO applications from quality assessments to gene prediction and phylogenomics. Mol. Biol. Evol. 35: 543-548.

Xia, P., Guo, H., Zhang, Y., Deyholos, M. K., Peng, L., Jia, Y., Yan, X., Liu, Y., and Liang, Z. 2016. Wild Panax vietnamensis and Panax stipuleanatus markedly increase the genetic diversity of Panax notoginseng (Araliaceae) revealed by start codon targeted (SCOT) markers and ITS DNA barcode. Biochem. Syst. Ecol. 66:37-42. 